\title{
Single-cell and spatial transcriptomics reveal somitogenesis in gastruloids
}

Susanne C. van den Brink ${ }^{\dagger^{*}}$, Anna Alemany ${ }^{\dagger^{\star}}$, Vincent van Batenburg ${ }^{{ }^{*}}$, Naomi Moris ${ }^{\#}$, Marloes Blotenburg $^{\dagger}$, Judith Vivié ${ }^{\dagger}$, Peter Baillie-Johnson ${ }^{\#,+}$, Jennifer Nichols ${ }^{+}$, Katharina F. Sonnen ${ }^{\S}$, Alfonso Martinez Arias $^{\#}$ \& Alexander van Oudenaarden ${ }^{\dagger}$

${ }^{\dagger}$ Oncode Institute, Hubrecht Institute-KNAW (Royal Netherlands Academy of Arts and Sciences) and University Medical Center Utrecht, 3584 CT, Utrecht, The Netherlands

\# Department of Genetics, University of Cambridge, Cambridge CB2 3EH, UK

+ Wellcome-Medical Research Council Cambridge Stem Cell Institute and Department of Physiology, Development and Neuroscience, University of Cambridge, Cambridge, UK

$\S$ Hubrecht Institute-KNAW (Royal Netherlands Academy of Arts and Sciences) and University Medical Center Utrecht, 3584 CT, Utrecht, The Netherlands

*These authors contributed equally: Susanne C. van den Brink, Anna Alemany, Vincent van Batenburg Correspondence: a.vanoudenaarden@hubrecht.eu (A.v.O.)

Gastruloids are three-dimensional aggregates of embryonic stem cells (ESCs) that display key features of mammalian post-implantation development, including germ layer specification and axial organization ${ }^{1-3}$. So far, the expression pattern of only a small number of genes in gastruloids has been explored with microscopy, but it is still unclear to what extent genome-wide expression patterns mimic those in embryos. Here, we compared mouse gastruloids with mouse embryos using single-cell RNA sequencing (scRNA-seq) and spatial transcriptomics (tomo-seq). We identify various embryonic cell types that were not known to be present in gastruloids, and show that key regulators of somitogenesis are expressed similarly between embryos and gastruloids. Using live-imaging we then show that the somitogenesis clock is active in gastruloids with dynamics resembling those in vivo. Since gastruloids can be grown in large quantities, we perform a small screen that revealed how reduced FGF signalling induces a short-tail phenotype in embryos. Finally, we demonstrate that Matrigel-embedding induces gastruloids to generate somites with correct rostral-caudal patterning, which appear sequentially in anterior to posterior direction over time. This study thus shows the power of gastruloids as a model system to explore development and somitogenesis in vitro in a high-throughput manner.

It has previously been shown that transcriptomes of entire gastruloids at 120 hours after aggregation $(120 \mathrm{~h})$ resembles that of E8.5 mouse embryos ${ }^{3}$. To extend this characterization to the single-cell level, we applied scRNA-seq to more than 25,000 cells obtained from 100 gastruloids (120 h) that were generated using either E14-IB10 or LfngT2AVenus mouse ESCs (Extended Data Fig. 1a-b, Methods), and clustered cells based on highly variable genes (Fig. 1a, Extended Data Fig. 1c-f, Supplementary Tables 1-2). To annotate the 13 resulting clusters, we compared their transcriptomes to a recently published scRNA-seq dataset from E8.5 mouse embryos ${ }^{4}$ (Fig. 1b, Methods, Supplementary Table 3). We confirmed the absence of anterior neuronal cell types and the presence of ectodermal cells 
resembling embryonic spinal cord ${ }^{1,3}$ (cluster 8; Extended Data Fig. 1g-h and 2). Additionally, we for the first time identified endothelial and haematoendothelial cells (cluster 10), and found a cluster with signatures of primordial germ cells and extra-embryonic ectoderm (cluster 12). Cluster 13 correlates with the visceral endoderm (VE); however, we suggest that this represents definitive endoderm (DE) since previous studies showed that VE has been incorporated into DE in E8.5 mouse embryos ${ }^{5,6}$. We find the olfactory receptor genes Olfr959 and Olfr129 upregulated in cluster 9, suggesting the presence of sensory neuron precursors. This cluster also expresses markers linked to head mesenchyme, pharyngeal pouches, branchial arches and neural crest and correlates with mesenchyme in embryos. Cluster 11 might represent allantoic cells, as it expresses Tbx4, which in E8.5 embryos is expressed exclusively in the allantois ${ }^{4,7}$. A comparison between both mouse ESC lines revealed that some cell types are more prevalent in one of the two lines (Extended Data Fig. 1e, Supplementary Tables 1,4), indicating that genetic background can skew the composition of gastruloids.

Many of the cells in gastruloids correspond to mesodermal subtypes, including neuromesodermal progenitors (NMPs), caudal, paraxial, somatic, pharyngeal and cardiac mesoderm (clusters 1-7; Fig. 1b). After careful examination, we concluded that the cells in clusters 1-8 are ordered along neural and mesodermal differentiation trajectories. To further explore this, we linearized the part of the UMAP containing clusters 1-8 (Methods) and plotted the expression of genes linked to neural and mesodermal differentiation processes along this linearized UMAP (Fig. 1c). First, we observed an NMP to neural differentiation trajectory from cluster 7 to 8 that starts with the expression of the tail bud genes $T$ (Brachyury), Nkx1-2, Cyp26a1 and that is followed by the expression of neural differentiation markers such as Sox2, Hes3, Sox 1 and $P a x 6^{8}$. Second, we observed a mesodermal differentiation trajectory from cluster 6 to 2. In good agreement with what happens in embryos, the expression levels of tail bud and Wnt/FGF signalling genes ( Fgf8, Fgf17 and Wnt3a) gradually decline in cells that differentiate towards a pre-somitic fate (characterized by the expression of $T b x 6$ and $H e s 7^{9}$ ), with expression levels being lower in the somite differentiation front (which expresses Ripply2). Upon somitic differentiation, cells first express Uncx4.1 and Tbx18, and later express markers more differentiated somites, such as Meox2 and $P a x 3^{9}$. Finally, cluster 1 expresses heart markers (Gata6 and $\mathrm{Hand} 2^{10}$ ).

In embryos, neural and mesodermal differentiation trajectories have a strong spatial component, with NMPs being located within the tail bud and differentiated tissues being located more anteriorly ${ }^{8}$. To determine whether the differentiation trajectories detected in gastruloids also have a spatial anteriorposterior (AP) component, we performed tomo-seq ${ }^{11}$ on 120 hours E14-IB10 and LfngT2AVenus gastruloids (Methods, Extended Data Fig. 3-5). For each cell line, we selected reproducible genes between replicates, and clustered these according to their AP expression pattern (Methods,

74 Supplementary Tables 5-6). The overall gene expression patterns between gastruloids generated from 75 the two ESC lines are similar (Fig. 1d, Extended Data Fig. 6, Supplementary Tables 6-8). To annotate the 76 various expression domains, we projected the mean expression of the genes in each tomo-seq cluster 77 onto the UMAP (Fig. 1e). This revealed that NMPs (cluster 7 in Fig. 1a and cluster II in Fig. 1e) are 
located in the most posterior tip of gastruloids. More differentiated neural cells are found slightly more anterior (Extended Data Fig. 3e). Furthermore, mesodermal clusters in the UMAP are sequentially ordered along the AP axis of gastruloids, with 6 being the most posterior and 2 the most anterior (cluster V-VIII in Fig. 1d-e; see also Extended Data Fig. 3e). This revealed that the neural and mesodermal differentiation trajectories in gastruloids are linked to their AP axis, which agrees with what occurs in embryos $^{8,9}$. Additionally, we found that the anterior domain in gastruloids (clusters VI-VIII) contains cardiac, endothelial and head mesenchymal cells (Fig. 3d-e, Extended Data Fig. 3e). This is consistent with the locations of these tissues in embryos.

To further investigate to what extent AP gene expression patterns in gastruloids recapitulate those in embryos, we applied tomo-seq to E8.5 embryos (Fig. 1f, Extended Data Figs. 3-6, Supplementary Tables 5-8 and Methods). This revealed that mesoderm genes and genes that regulate somitogenesis, are expressed very similarly between embryos and gastruloids. We detected cardiac and brain domains in embryos (cluster VII and I in Extended Data Figure 5b, respectively) that are not clearly defined and absent, respectively, in gastruloids. We found additional differences and similarities between embryos and gastruloids that are presented in detail in the supplement (Extended Data Fig. 5 and Supplementary Tables 7-8; for visualization, see https://avolab.shinyapps.io/962095337353856/). We also compared our gastruloid tomo-seq dataset to a previously published microarray dataset where the posterior mesoderm (from the tail bud to the newly formed somite) of E9.5 mouse embryos was dissected $^{12}$ (Fig. 1g, Extended Data Figs. 4-5 and Supplementary Tables 5-8). This comparison reveals a striking similarity between gastruloids and the mesoderm of embryos.

In embryos, the organization of the mesoderm is established by dynamic gene regulatory networks that are tightly linked to the process of somitogenesis ${ }^{9}$. During somitogenesis, AP retinoic acid and opposing Wnt/FGF signalling gradients determine the position of the differentiation front, which induces the differentiation of the mesoderm into epithelial blocks called somites (Fig. 2a). These somites have defined rostral and caudal halves, and appear sequentially in AP direction. During this process, the tail bud of the embryo grows, and consequently, the signalling gradients and differentiation front move posteriorly over time. A second component of somitogenesis entails oscillations of Wnt, Notch and FGF signalling, where signalling waves travel from the tail bud towards the differentiation front every $\sim 2$ hours in mice ${ }^{9,13}$. This cyclic component of the somitogenesis process is known as the "segmentation clock" and is thought to regulate the timing of somite formation ${ }^{9,14}$. To investigate whether the segmentation clock is active in gastruloids, we monitored Notch signalling activity by performing fluorescence time-lapse imaging on gastruloids generated from LnfgT2AVenus mouse $\mathrm{ESCs}^{15}$ (Methods). Similar to what has been seen in embryos ${ }^{15}$, we observed a dynamic differentiation front, which expresses high levels of $L$ fng and regresses posteriorly as the gastruloids extend (Fig. 2b, Extended Data Fig. 7-8, Supplementary Video 1). Additionally, we observed oscillating waves with low expression of $L$ fng and a period of about 2 114 The expression of $L$ fng disappears in the presence of the Notch inhibitor DAPT (Extended Data Fig. 7, 
Supplementary Video 2), confirming that the reporter expression is dependent on Notch signalling in gastruloids, as it is in embryos ${ }^{16}$. These experiments indicate that the segmentation clock is active in gastruloids with dynamics that are very similar to the in vivo situation.

Gastruloids can be easily generated in large numbers, opening the possibility to perform screens.

To exemplify this, we performed a small compound screen on LfngT2AVenus gastruloids and investigated the effect of inhibitors and agonists of FGF, Wnt, and BMP signalling pathways on the speed of the differentiation front (Supplementary Videos 3, Extended Data Figs. 7 and 8e-f). This revealed that the application of the MEK/ERK pathway inhibitor PD03, which inhibits FGF signalling, speeds up the differentiation front in a dose-dependent manner without altering the speed by which gastruloids grow posteriorly (Fig. 2f, Extended Data Fig. 9a, Supplementary Video 4). This imbalance between the speed of the differentiation front and gastruloid growth results in a progressive decrease in the length of the presomitic mesoderm, and in gastruloids that stop growing prematurely (Fig. 2g). Similar results were obtained with the FGF receptor inhibitors PD17 and BGJ398 (Extended Data Figs. 7 and 8f, Supplementary Video 5). Our observations provide an explanation for the observed short-tail phenotype of FGF-mutant mouse embryos ${ }^{17}$ and posteriorly shifted differentiation fronts after FGF inhibition ${ }^{18,19}$.

Even though our experiments reveal that key regulators of somitogenesis are expressed in the correct location and that the segmentation clock is active in gastruloids, gastruloids that are generated with previously published protocols do not form somites ${ }^{1,3,20}$. Remarkably, during our real-time imaging experiments, we occasionally observed small "indentations" that appeared anteriorly to the differentiation front (Supplementary Video 4). These segments were only visible in gastruloids mounted in Matrigel at 96 $\mathrm{h}$, which was done prior to the real-time imaging experiments to stabilize them (Methods). We then performed in situ hybridization (ISH) stainings for Uncx4.1 (a marker for the caudal halves of somites ${ }^{9}$; Fig. 2a) and found that Uncx4.1 was expressed in a stripy pattern in $4 \%$ (4 out of 100) of the $120 \mathrm{~h}$ gastruloids that were embedded in 100\% Matrigel at $96 \mathrm{~h}$ (Fig. 3a). Such a pattern was never detected in $120 \mathrm{~h}$ gastruloids cultured without Matrigel. To explore the effect of the concentration of Matrigel, we performed a titration experiment. We found that embedding $96 \mathrm{~h}$ gastruloids in 10-25\% Matrigel resulted in the formation of clear segments of which the posterior half is marked by Uncx4.1 expression in up to 143 Fig. 9b). Time-lapse imaging movies on these gastruloids revealed that the segments appear sequentially 144 in AP direction, anteriorly to the Lfng expression domain (Fig. 3c, Supplementary Video 6 and Extended somite) and for Uncx4.1 and Tbx18 (a marker of rostral somites ${ }^{9}$ ) revealed that Uncx4.1 and Tbx18 are expressed in an alternating pattern (Fig. 3d), and it is indeed the caudal half of the segments that expresses Uncx4.1 (Extended Data Fig. 10). At $120 \mathrm{~h}$ of culture (after $24 \mathrm{~h}$ in 10\% Matrigel), gastruloids have $\sim 10-11$ somites (Fig. 3d, Extended Data Fig. 10), whose size decreases in the AP direction, from on average 183 to $43.4 \mu \mathrm{m}$ (Extended Data Fig. 10c-e). In embryos, the size of these somites decreases from 120 to $80 \mu \mathrm{m}$ (Methods). Our experiments thus reveal that embedding gastruloids in low-percentage 
Matrigel induces the formation of somites, which have correct rostral-caudal patterning and appear sequentially along the AP direction over time. We have so far not observed gastruloids with two neighbouring rows of somites, and it will be interesting to explore why this is the case in future studies. Using single-cell and spatial transcriptomics we demonstrate that gene expression in murine gastruloids is very similar to embryos. Gastruloids can therefore be used as a model system for embryology, and have some key advantages over embryos: they can be grown in large quantities

158 allowing screens, are easier to genetically modify as they can be grown directly from ESCs, and can be 159 used to study human development (see accompanying manuscript ${ }^{22}$ ). We utilized several of these 160 advantages to study somitogenesis in vitro. Recent pioneering studies have explored ex vivo and in vitro 161 models for somitogenesis, such as monolayer-PSM cultures ${ }^{23,16}$ and cultures of embryoid body-like 162 aggregates of mouse ESCs that display travelling somitogenesis waves in vitro ${ }^{24}$. However, such cultures 163 do not form proper somites, lack a correctly defined AP axis and do not elongate in posterior direction. 164 Here, we have shown that gastruloids overcome these limitations, and thus provide a powerful tool to 165 study somitogenesis in vitro. In general, in vitro mimics of development, such as gastruloids, are 166 promising systems with which we are starting to obtain new insights that could not readily be obtained 167 with embryos. We therefore anticipate many applications of this system, which will aid to unravel the 168 complex processes that regulate embryogenesis. 


\section{References}

1. van den Brink, S. C. et al. Symmetry breaking, germ layer specification and axial organisation in aggregates of mouse embryonic stem cells. Development 141, 4231-4242 (2014).

2. Turner, D. A. et al. Anteroposterior polarity and elongation in the absence of extra-embryonic tissues and of spatially localised signalling in gastruloids: mammalian embryonic organoids. Development 144, 3894-3906 (2017).

3. Beccari, L. et al. Multi-axial self-organization properties of mouse embryonic stem cells into gastruloids. Nature 562, 272-276 (2018).

4. Pijuan-Sala, B. et al. A single-cell molecular map of mouse gastrulation and early organogenesis. Nature 566, 490-495 (2019).

5. Nowotschin, S. et al. The emergent landscape of the mouse gut endoderm at single-cell resolution. Nature 569, 361-367 (2019).

6. Kwon, G. S., Viotti, M. \& Hadjantonakis, A. K. The endoderm of the mouse embryo arises by dynamic widespread intercalation of embryonic and extraembryonic lineages. Dev. Cell 15, 509-520 (2008).

7. Scotti, M. \& Kmita, M. Recruitment of 5' Hoxa genes in the allantois is essential for proper extraembryonic function in placental mammals. Development 139, 731-739 (2012).

8. Koch, F. et al. Antagonistic activities of Sox2 and Brachyury control the fate choice of neuromesodermal progenitors. Dev. Cell 42, 514-526.e7 (2017).

9. Chal, J. \& Pourquié, O. Making muscle: skeletal myogenesis in vivo and in vitro. Development 144, 2104-2122 (2017)

10. Laurent, F. et al. HAND2 Target Gene Regulatory Networks Control Atrioventricular Canal and Cardiac Valve Development. Cell Rep. 19, 1602-1613 (2017).

11. Junker, J. P. et al. Genome-wide RNA Tomography in the zebrafish embryo. Cell 159, 662-675 (2014).

12. Chal, J. et al. Differentiation of pluripotent stem cells to muscle fiber to model Duchenne muscular dystrophy. Nat. Biotechnol. 33, 962-969 (2015).

13. Dequeant, M. L. et al. A complex oscillating network of signaling genes underlies the mouse segmentation clock. Science 314, 1595-1598 (2006). 
14. Oates, A. C., Morelli, L. G. \& Ares, S. Patterning embryos with oscillations: structure, function and dynamics of the vertebrate segmentation clock. Development 139, 625-639 (2012).

15. Sonnen, K. F. et al. Modulation of phase shift between Wnt and Notch signaling oscillations controls mesoderm segmentation. Cell 172, 1079-1090 (2018).

16. Hubaud, A., Regev, I., Mahadevan, L. \& Pourquié, O. Excitable dynamics and Yap-dependent mechanical cues drive the segmentation clock. Cell 171, 668-682 (2017).

17. Naiche, L. A., Holder, N. \& Lewandoski, M. FGF4 and FGF8 comprise the wavefront activity that controls somitogenesis. Proc. Natl. Acad. Sci. U. S. A. 108, 4018-4023 (2011).

18. Dubrulle, J., McGrew, M. J. \& Pourquié, O. FGF signaling controls somite boundary position and regulates segmentation clock control of spatiotemporal Hox gene activation. Cell 106, 219-232 (2001).

19. Sawada, A. et al. Fgf/MAPK signalling is a crucial positional cue in somite boundary formation. Development 128, 4873-4880 (2001).

20. Baillie-Johnson, P., van den Brink, S. C., Balayo, T., Turner, D. A. \& Martinez Arias, A. Generation of aggregates of mouse embryonic stem cells that show symmetry breaking, polarization and emergent collective behaviour in vitro. J. Vis. Exp. (2015).

21. Choi, H. M. T. et al. Third-generation hybridization chain reaction: multiplexed, quantitative, sensitive, versatile, robust. Development 145, 1-10 (2018).

22. Moris, N. et al. An embryonic stem cell-based model for early human development. Unpublished

23. Lauschke, V. M., Tsiairis, C. D., François, P. \& Aulehla, A. Scaling of embryonic patterning based on phase-gradient encoding. Nature 493, 101-105 (2013).

24. Matsumiya, M., Tomita, T., Yoshioka-Kobayashi, K., Isomura, A. \& Kageyama, R. ES cell-derived presomitic mesoderm-like tissues for analysis of synchronized oscillations in the segmentation clock. Development 145, (2018).

\section{Extended data}

172 Ten Extended Data Figures, nine Supplementary Tables and six Supplementary Videos are available for 173 this publication. 


\section{Acknowledgements}

176 This work was supported by an European Research Council Advanced grant (ERC-AdG 742225177 IntScOmics), a Nederlandse Organisatie voor Wetenschappelijk Onderzoek (NWO) TOP award (NWO178 CW 714.016.001), and the Foundation for Fundamental Research on Matter, financially supported by 179 NWO (FOM-14NOISE01) to S.C.v.d.B., A.A., V.v.B., M.B., J.V. and A.v.O., a BBSRC (No. BB/M023370/1 and BB/P003184/1), Newton Trust (INT16.24b) and MRC (MR/R017190/1) grant to A.M.A., a Newnham College Cambridge Junior Research Fellowship to N.M., and a studentship from the Engineering and Physical Sciences Research Council (EPSRC) to P.B.J.. The Cambridge Stem Cell Institute is supported by core funding from the Wellcome Trust and Medical Research Council; J. N. was funded by the University of Cambridge, and K.F.S. by core funding from the Hubrecht Institute. This work is part of the Oncode Institute which is partly financed by the Dutch Cancer Society. We thank A. Ebbing and M. Betist for the robotized tomo-seq protocol, G. Keller for the Brachyury ${ }^{G F P}$ cell line, J. Collignon for the Nodal ${ }^{Y F P}$ line, K. Hadjantonakis for the TCF/LEF ${ }^{m C h e r r y}$ line, S. van den Brink and E. R. Maandag for the E14-IB10 cells and J. Kress and A. Aulehla for the LfngT2AVenus mouse ESC line. In addition, we thank I. Misteli Guerreiro, J. Peterson-Maduro and J. Hoeksma for suggestions for ISH experiments and W. Thomas, Y. el Azhar, J. Juksar, and J. Beumer for reagents and inhibitors. We also thank A. de Graaff from the Hubrecht Imaging Facility and A. Stokkermans for help with multi-photon microscopy and analysis of the microscopy data, D.A. Turner for microscopy panels that were used for tomo-seq validation, J. Korving for help with the somite-size measurements in embryos, the Hubrecht FACS facility and R. van der Linden for FACS experiments, Single Cell Discoveries for 10x Genomics scRNA-seq, the Utrecht Sequencing facility for sequencing and P. Zeller, H. Viñas Gaza and all members of the van Oudenaarden and Martinez Arias laboratories for discussions.

\section{Author contributions}

S.C.v.d.B. and A.v.O. conceived and designed the project. S.C.v.d.B. and V.v.B. generated gastruloids, and S.C.v.d.B., M.B. and J.V. performed scRNA-seq experiments. Embedding of mouse gastruloids for tomo-seq was done by S.C.v.d.B.; N.M. and P.B.J. embedded mouse embryos for tomo-seq with help from J.N.. S.C.v.d.B. cryosectioned gastruloids and embryos and performed tomo-seq experiments, and J.V. developed the robotized tomo-seq protocol. A.A. performed the mapping and analysis, including comparisons with embryonic datasets, of the scRNA-seq and tomo-seq data. A.v.O. performed the linearized UMAP analysis. S.C.v.d.B., M.B., A.A., N.M. and A.M.A. interpreted the sequencing datasets. P.B.J. performed the first Matrigel-embedding pilot experiments. V.v.B. performed time-lapse imaging experiments, ISH and HCR stainings, with help from S.C.v.d.B. and K.F.S.. V.v.B. analysed the microscopy data, with support from K.F.S., and V.v.B., S.c.v.d.B., A.v.O. and K.F.S. interpreted the imaging results. S.C.v.d.B., A.A., V.v.B. and A.v.O. wrote the manuscript with support from K.F.S. and 


\section{Author information}

213 Susanne C. van den Brink, Anna Alemany and Vincent van Batenburg contributed equally to this work.

214

215 Data availability

216 All RNA-seq datasets produced in this study are deposited in the Gene Expression Omnibus (GEO)

217 under accession code GSE123187. All the scripts used to analyse the data are freely available upon

218 request. All scRNA-seq and tomo-seq data can be explored at

219 https://avolab.shinyapps.io/962095337353856/.

220

221 Competing interests

222 There are no competing interests for this work.

223

224 Correspondence

225 Correspondence to Alexander van Oudenaarden (a.vanoudenaarden@hubrecht.eu). 
Fig. 1 | scRNA-seq and tomo-seq on mouse gastruloids and comparison to embryos. a, Uniform manifold approximation and projection (UMAP) plot showing cells isolated from $120 \mathrm{~h}$ gastruloids (26 and 74 gastruloids grown using E14-IB10 and LfngT2AVenus ${ }^{15}$ ESC lines, respectively) cultured in standard $^{1,20}$ conditions. Cells are coloured and numbered by their cluster annotation. $\mathbf{b}$, Dot plot showing overlapping genes between significantly upregulated genes for each gastruloid cluster and each E8.5 mouse embryonic cell type ${ }^{4}$. Dot colour indicates the probability of finding such a number of overlapping genes between the two sets by random chance ( $P$-value). Dot size represents the number of overlapping genes. c, Linearized UMAP of clusters 1-8 (top) and expression profiles of genes related to neural and mesodermal differentiation ${ }^{8,9}$ (bottom). Green and grey shades indicate location of cardiac cells and NMPs, respectively. The position of each cell along the $x$-axis relates to its differentiated state towards a neural or mesodermal fate. d, Heatmap showing the average AP expression pattern of 514 genes detected by tomo-seq ${ }^{11}$ in $120 \mathrm{~h}$ gastruloids generated from E14-IB10 and LfngT2AVenus ${ }^{15}$ mouse ESCs using standard ${ }^{1,20}$ culture protocols. Only genes reproducible between all replicates of E14-IB10 $(n=5)$ and LfngT2AVenus $(n=3)$ gastruloids are shown. Genes are clustered based on AP expression pattern (Supplementary Tables 5-6); Roman-numbered bars represent tomo-seq clusters. e, Mean log expression of genes present in each tomo-seq cluster plotted on the UMAP. f, $\mathbf{g}$, As in d, but showing 222 genes (f) or 239 genes (g) found reproducible between replicates of E14-IB10 and LfngT2AVenus gastruloids, and (f) E8.5 mouse embryos ( $n=3$ ); or ( $g$ ) posterior mesoderm of E9.5 mouse embryos ${ }^{12}$ MD, mesoderm; ExE, extra-embryonic; EcD, ectoderm; EnD, endoderm; PGC, primordial germ cells; prog, progenitors; Haemato, haemato-endothelial; NMP, neuro-mesodermal progenitors; PSM, presomitic mesoderm; E14, E14-IB10; Lfng, LfngT2AVenus.

Fig. 2 | Real-time imaging and perturbation of the segmentation clock in mouse gastruloids. a, Illustration of somitogenesis in mouse embryos. Dark blue, retinoic acid (RA) gradient; red area and arrows, dynamic expression of Lfng; green, FGF/Wnt signalling gradient in PSM (presomitic mesoderm); magenta/cyan blocks, somites; blocks with dotted lines, newly forming somites; posterior dotted line, posterior elongation of the PSM. b, Real-time imaging of a LfngT2AVenus ${ }^{15}$ gastruloid embedded in $100 \%$ Matrigel at $96 \mathrm{~h}$ and subsequently imaged for 17 hours (Supplementary Video 1). Blue arrowheads show the AP displacement of the differentiation front (Lfng expressing; red). c, Kymograph along the AP axis of a LfngT2AVenus gastruloid embedded in $100 \%$ Matrigel at $96 \mathrm{~h}$ and subsequently imaged for 30 h. Highest intensity signal reflects the posteriorly moving differentiation front (blue arrowhead in b); white arrowheads indicate periodic oscillations in the PSM. d, Detrended LfngT2AVenus intensity along the dashed white line in d. A.U., arbitrary units. e, Periodogram of the Lfng oscillations detected in 13 LfngT2AVenus gastruloids, as determined by Lomb-Scargle decomposition (Methods). $\mathbf{f}$, Speed of elongation and differentiation front in LfngT2AVenus gastruloids treated with PD03. Box plots: center line, median; box limits, 1st and 3rd quartiles; whiskers, range. Each point is one replicate. g, Illustration 
explaining the effect of FGF inhibition, which increases the speed of the differentiation front (red arrows, $V_{\text {Diff }}$ ) without altering the elongation rate (blue arrows, $\left.V_{P S M}\right)$ of gastruloids. Three timepoints $\left(t_{1}, t_{2}, t_{3}\right)$ are depicted. White tissue, non-differentiated tissue (PSM), grey tissue, differentiated tissue; A, anterior; P, posterior; scale bar, $200 \mu \mathrm{m}$.

Fig. 3 | Stainings and real-time imaging of somite formation in gastruloids embedded in low percentages of Matrigel. a, ISH staining for Uncx4.1 on $120 \mathrm{~h} \mathrm{LfngT2AVenus} \mathrm{gastruloids} \mathrm{that} \mathrm{were} \mathrm{not}$ embedded in Matrigel ( $0 \%$; standard, previously published protocol ${ }^{1,20}$ ) or that were embedded in $25 \%$ or $100 \%$ Matrigel at $96 \mathrm{~h}$. Numbers below panels indicate number of gastruloids where stripy Uncx4.1 expression patterns were observed. $\mathbf{b}$, Somites in a LfngT2AVenus gastruloid (zoomed in; Extended Data Fig. 9b) embedded in $10 \%$ Matrigel at $96 \mathrm{~h}$ and stained for Uncx4.1 using $\mathrm{HCR}^{21}$ at $120 \mathrm{~h}$. Magenta arrowheads, segment boundaries. c, Real-time imaging (Supplementary Video 6) of LfngT2AVenus gastruloids embedded in 10\% Matrigel at $96 \mathrm{~h}$. Blue arrowheads, differentiation front (Lfng expressing, red); magenta arrowheads, appearing segment boundaries. $\mathbf{d}, \mathrm{HCR}^{21}$ double staining for Uncx4.1 (cyan) and Tbx18 (magenta) (Fig. 2a) ${ }^{9}$, on a $120 \mathrm{~h}$ LfngT2AVenus gastruloid embedded in $10 \%$ Matrigel at $96 \mathrm{~h}$ and to which $1.3 \mu \mathrm{M}$ of PD03 was added at $96.5 \mathrm{~h}$. White asterisks mark Uncx4.1 expression stripes. A, Anterior; P, Posterior; scale bar in panels a and d: $200 \mu \mathrm{m}$; scale bar in panels b and c, $100 \mu \mathrm{m}$.

\section{Methods}

Mouse gastruloid culture, with and without Matrigel. E14-IB10 (subclone of 129/Ola-derived E14 ES cells from The Netherlands Cancer Institute), LfngT2AVenus ${ }^{15}$ (Notch-signalling reporter; contains a single copy of Venus that was inserted in the endogenous $L$ fng locus ${ }^{15}$; the selection cassette was removed), Brachyury ${ }^{G F P(25)}$, Wnt/ $\beta$-catenin transcriptional reporter $T C F / L E F^{m C h e r r y(26,27)}$ and Nodal ${ }^{Y F P(28)}$ mouse ESCs were maintained in standard conditions in serum + LIF (ESLIF medium) on gelatinized 6well plates and in a humidified incubator $\left(5 \% \mathrm{CO}_{2}, 37^{\circ} \mathrm{C}\right)$ as described before $\mathrm{C}^{20,26,29-32}$. Gastruloids for scRNA-seq and tomo-seq experiments were generated as described previously ${ }^{1,20}$, with the following minor modifications: after neutralization of trypsin with ESLIF, cells were washed with PBS (containing $\mathrm{Ca} 2+$ and $\mathrm{Mg} 2+$ ) twice. Next, cells were resuspended in N2B27 medium (NDiff 227 medium, Takara, Y40002), and the cell concentration was determined only after resuspension in N2B27 medium. Cells were then diluted in N2B27 to a concentration of 7.5 cells $/ \mu \mathrm{l}$, and $40 \mu \mathrm{l}$ (with $\sim 300$ cells) of this suspension was transferred to each well of a U-bottomed 96-well plate (Greiner Bio-One, 650185). N2B27 aliquots were stored at $-20^{\circ} \mathrm{C}$ and thawed by rocking them at $4{ }^{\circ} \mathrm{C}$ for several hours, after which aliquots were transferred to a cell culture flask in a $\mathrm{CO}_{2}$-controlled $37^{\circ} \mathrm{C}$ incubator for $\mathrm{pH}$-equilibration one day before gastruloid formation. Aggregates that did not elongate and that did not form gastruloids were excluded from this study, and curved gastruloids were excluded from tomo-seq experiments. For the scRNA-seq and tomo-seq experiments, $120 \mathrm{~h}$ gastruloids generated with the original gastruloids protocol $^{1,20}$ were used, as these gastruloids were in our hands more reproducible (significantly less 
variation in morphology between wells) than more recent versions of the protocol, that allow culture up to $168 \mathrm{~h}^{3}$. For ISH and HCR staining and real-time imaging experiments, gastruloids were cultured as described above, but then embedded in Matrigel at $96 \mathrm{~h}$. To embed gastruloids in $50-100 \%$ Matrigel (Corning, 356231, lot number 6137007, protein concentration $9.8 \mathrm{mg} / \mathrm{mL}$ ), Matrigel was thawed on ice, mixed with the required amount of cold N2B27 medium, and $60 \mu \mathrm{l}$ was added to each well of a multi-well imaging chamber (Sigma, EP0030741021 or M9312) on ice. $96 \mathrm{~h}$ gastruloids were then transferred to the Matrigel using a $20 \mu \mathrm{l}$ pipet and allowed to settle for approximately $5 \mathrm{~min}$ before the chamber was incubated at $37{ }^{\circ} \mathrm{C}$ for $10 \mathrm{~min}$, allowing the Matrigel to solidify. After this, $500 \mu \mathrm{L}$ N2B27 medium was added to each well. Embedding gastruloids in diluted 10-25\% Matrigel was done by first pooling the gastruloids in a $5 \mathrm{~mL}$ low binding Eppendorf tube on ice, replacing the N2B27 medium with fresh cold medium and then adding the correct volume of Matrigel. The gastruloids were then transferred to a 24 wells plate (Sigma, EP0030741021 or M9312) using a p1000 pipet with the tip cut off, at a concentration of $\sim 8$ gastruloids per $\mathrm{mL}, 500 \mu \mathrm{L}$ per well.

Dissociation and FACS of gastruloids prior to scRNA-seq. To dissociate gastruloids for scRNA-seq, gastruloids were washed with PBS $2 x$, incubated in Trypsin-EDTA at $37{ }^{\circ} \mathrm{C}$ for 5 min and titrated with a p200 pipette, after which ESLIF (see above) was added to neutralize the Trypsin. After centrifugation (170g, $3 \mathrm{~min}$ ), cells were resuspended in PBS with 10\% serum and filtered through a $35 \mu \mathrm{m}$ filter (Falcon, 352235). Prior to FACS, DAPI (Thermo Fisher) was added to assess cell viability. For SORT-seq, individual live cells were sorted into the wells of a 384-well plate as described previously ${ }^{33}$ using a BD FACSJazz ${ }^{\text {TM }}$ Cell Sorter (BD Biosciences) that was equipped with BD FACS software (version 1.2.0.124). For 10x Genomics scRNA-seq, washes were done using PBS0 (PBS without calcium and magnesium), and 100,000 live cells were sorted into $1.5 \mathrm{ml}$ DNA lowbind tubes (Eppendorf, 022431021) that were prefilled with $50 \mu \mathrm{l} \mathrm{PBS0}$, after which cells were centrifuged for 3 min at $200 \mathrm{~g}$, resuspended in $80 \mu \mathrm{l}$ PBS0 containing $5-10 \%$ serum, and filtered through a $35 \mu \mathrm{m}$ filter (Falcon, 352235). After resuspension and filtering, the cell concentration was determined using a counting chamber (Bürker-Türk, Marienfeld). scRNA-seq (SORT-seq and 10x Genomics). For scRNA-seq, cells extracted from $120 \mathrm{~h}$ gastruloids (120 h; generated with a previously published, non-Matrigel based protocol ${ }^{1,20}$ ) were processed using either SORT-seq (CEL-seq2 based scRNA-seq on cells that were sorted into 384-well plates ${ }^{33}$ ) or using the 10x Genomics Chromium Single Cell 3' ( $v 3$ Chemistry) gene expression kit, according to manufacturer's instructions.

Animal experimentation. Mouse embryos $(n=3)$ used for tomo-seq were derived from crosses between CD-1 females and CD-1 stud males. Experiments were performed in accordance with EU guidelines, under the authority of appropriate UK governmental legislation. Use of animals for this project was approved by the Animal Welfare and Ethical Review Body for the University of Cambridge. Relevant Home Office licenses are in place.

337 Tomo-seq. Tomo-seq was performed using a robotized (SORT-seq ${ }^{33}$ based) version of a previously 338 published tomo-seq protocol ${ }^{11}$. Briefly, $120 \mathrm{~h}$ gastruloids ( $\mathrm{n}=3 \mathrm{E} 14-\mathrm{IB} 10$ gastruloids sectioned using 20 
$\mu \mathrm{m}$ sections; $\mathrm{n}=2$ E14-IB10 gastruloids sectioned using $8 \mu \mathrm{m}$ sections; $\mathrm{n}=3$ LfngT2AVenus gastruloids sectioned using $20 \mu \mathrm{m}$ sections, generated with previously published, non-Matrigel based gastruloid protocols ${ }^{1,20}$ ) or E8.5 mouse embryos ( $n=3$ sectioned using $20 \mu \mathrm{m}$ sections) were embedded in cryosolution (Leica, 14020108926), snap-frozen on dry-ice, stored at $-80{ }^{\circ} \mathrm{C}$ and sectioned using a cryotome. Sections were collected in the wells of a Hard-Shell PCR Low-profile, semi-skirted 96-well plate (Bio-rad, HSL9601) that was already prefilled with mineral oil (Sigma, M8410-1L) and CEL-seq2 primers. For each well, a unique, barcoded CEL-seq2 primer was used, which allowed us to pool the content of the wells after second strand synthesis. To sequence the mRNA content of the wells, SORT-seq (robotized CEL-seq2 based scRNA-seq ${ }^{33}$ ) was performed using a Nanodrop II liquid handling platform (GC biotech).

Sequencing. Sequencing was performed on the Illumina Next-seq sequencing platform. For SORT-seq and tomo-seq, paired end (75 bp) sequencing was performed; for 10x Genomics, sequencing was performed according to 10x Genomics manufacturer's instructions (Read1, 28 cycles; Index i7, 8 cycles; Read2, 91 cycles).

Mapping sequencing data. For SORT-seq and tomo-seq, the first 6 bases of read 1 contain the unique molecular identifier (UMI) and the next 7 bases contain the cell or section barcode. For 10x Genomics, the first 16 bases of read 1 contain the cell barcode, and the next 12 contain the UMI. For all sequencing experiments, read 2 contains the biological information. Reads 2 with a valid cell/section barcode were selected, trimmed using TrimGalore-0.4.3 with default parameters, and mapped using STAR-2.5.3a with default parameters to the mouse mm10 genome (Ensembl 93). Only reads mapping to gene bodies (exons or introns) were used for downstream analysis. Reads mapping simultaneously to an exon and to an intron were assigned to the exon. For each cell or section, the number of transcripts was obtained as previously described ${ }^{34}$. We refer to transcripts as unique molecules based on UMI correction. Mappabilities for both scRNA-seq and tomo-seq experiments range from $35 \%$ to $60 \%$. Spike-ins, ribosomal, and mitochondrial genes were removed from downstream analysis, together with Kcnq1ot1, Mir5109, Lars2, Malat1, Rn45s, because these genes seem to be linked to mapping errors and have been shown to be erroneous in earlier studies ${ }^{34}$.

Processing single-cell data. scRNA-seq analysis was performed using the Scanpy package ${ }^{35}$ (v1.4.3). In each experiment, cell barcodes with more than 1,000 transcripts and fewer than 6,000 genes were selected. Genes detected in fewer than 3 cells were excluded. Expression levels for each cell were sizenormalized to 10,000 transcripts. Highly variable genes were defined as those with a mean expression value between 0.0125 and 5 , and with a minimum dispersion, and used to generate the UMAPs shown in Fig. 1 and Extended Data Figs. 1, 2, 4, 5. Next, cells from the three independent experiments were analysed together. Here, we kept cells with more than 700 and fewer than 8,000 genes, and more than 1,000 and fewer than 40,000 transcripts. Selection of highly variable genes and cell normalization were performed as described above. To remove batch effects, we used the combat function from Scanpy (a Python implementation (https://github.com/brentp/combat.py) of the R-package Bioconductor ${ }^{36,37}$ ). Cells 
were clustered using a combination of k-medoids and Leiden algorithms ${ }^{38}$ (Supplementary Table 1). Differentially expressed genes in each cluster were determined using the t-test (Supplementary Table 2).

378 Comparison between gastruloid cell types and mouse embryonic cell types. Common genes between marker genes detected in the gastruloid cell clusters (Supplementary Table 1, $P$-value $<0.01$ and $\log 2$ (fold-change) > 1.01) and markers genes found for the different embryonic cell types defined in a previously published mouse embryo scRNA-seq dataset ${ }^{4}$ were found. $P$-value for significance was assigned using a binomial test, where the probability of sharing a number of common marker genes between a gastruloid cell type and an embryonic cell type was determined by randomizing the list of marker genes for the embryonic cell type from the full list of marker genes in the embryonic cell types ( $n=$ 200). Fig. 1 only shows comparison to embryonic cell types found at E8.5. Extended Data Fig. 1h shows the comparison to all embryonic cell types detected from E7.0 until E8.5. Only embryonic cell types with at least one cluster comparison with a $P$-value below 0.2 are shown. Using different $P$-value thresholds to define up-regulated genes does not have a significant impact on the results of the comparison between gastruloid cell populations and embryonic cell types.

Linearization of the UMAP. Cells in clusters 1-8 were projected on the symmetry axis along the clusters 1-8 in the UMAP (Extended Data Fig. 1d). The position of each cell along this symmetry axis defines the $\mathrm{x}$-position in Fig. 1c. To plot gene expression along the linearized UMAP, 1,000 evenly spaced bins were defined along the $x$-axis for which the expression average of all cells per respective bin was scaled and plotted. For visualization, a LOESS smoother was used with span set to 0.2 .

Processing tomo-seq data. $20 \mu \mathrm{m}$ sectioned slides with fewer than 3,200 genes and $8 \mu \mathrm{m}$ sectioned slices with fewer than 6,000 genes were filtered out (Extended Data Fig. 3). In each tomo-seq sample, data was normalized to the median number of unique transcripts per slide. Sequencing libraries contain a maximum of 96 slices. In samples with more than 96 sections, several libraries were generated. For these samples, we corrected batch effects between sequenced libraries by imposing the continuity of expression profiles along the AP axis for each gene separately.

Gene reproducibility analysis between replicates. The Pearson correlation coefficient between the AP expression pattern (in z-score units) of two different samples is computed for all possible pairs of replicates. Linearly interpolated gene expression profiles are used when the number of sections is different between replicates. To assess for significant correlations, we randomly generate 10,000 expression profiles with the same number of sections as in the pair of replicates and determine a threshold for the correlation value at which less than $n$ random profiles have larger correlation values $(n=$ 100 for $P$-value $<0.01 ; n=500$ for $P$-value $<0.05$, etc; Supplementary Table 5). Only genes that are significantly correlated $(P$-value $<0.01)$ in at least five possible pairs of replicates are considered as reproducible between replicates (Supplementary Tables 6 and 9). Custom made code was used for this analysis.

Clustering genes based on AP expression patterns. Genes were first clustered based on z-score AP expression pattern using self-organizing maps with an initial number of clusters set to $\sim 5 \sqrt{ } N$, where $N$ is 
413 the total number of genes. Average z-score expression patterns for each cluster were then hierarchically 414 clustered using Euclidean distances and the Wart.D method.

415 Comparison between tomo-seq data of mouse embryos and mouse gastruloids. Gene 416 reproducibility analysis between the individual replicates of the systems that are being compared are 417 performed independently, as described above (Supplementary Tables 5-9). For heatmaps in Fig. 1d, f-g, 418 only genes present in the two separate lists of significantly correlated genes are used for downstream 419 analysis (Supplementary Tables 7 and 9). For heatmaps in Extended Data Fig. 5, genes that were 420 present in only one of the two separate lists were included as well (Supplementary Tables 8-9). Genes 421 were clustered based on their AP expression pattern in the systems that are being compared 422 simultaneously, as described above. The Pearson correlation coefficient for each gene is calculated 423 between the AP expression pattern of two different samples (in z-score units). To assess for significantly 424 correlated genes, we randomly generate 10,000 expression profiles with the same number of sections as 425 in the pair of replicates and determine the correlation value at which less than 500 random profiles have 426 larger correlation values $(P$-value $<0.05)$.

427 Comparison between genes in tomo-seq clusters and mouse embryonic cell types. As above, but 428 then calculating the number of overlapping genes, and the $P$-value of this overlap, by comparing the 429 genes in each tomo-seq cluster with the list of genes upregulated in the cell types of a previously 430 published E8.5 mouse embryo scRNA-seq dataset ${ }^{4}$ (Supplementary Tables 5-9).

431 Wide field microscopy. Widefield images of gastruloids made from Brachyury ${ }^{\text {GFP }}{ }^{25}$, Nodal ${ }^{\text {YFP } 28}$ and 432 TCF/LEF ${ }^{\text {mCherry }}\left(\right.$ TLC2 ${ }^{26,27}$ ) mouse ESCs were acquired at $120 \mathrm{~h}$ using a Zeiss AxioObserver Z1 in a 433 humidified $\mathrm{CO}_{2}$ incubator $\left(5 \% \mathrm{CO}_{2}, 37^{\circ} \mathrm{C}\right)$ and a $20 \mathrm{x}$ LD Plan-Neofluar $0.4 \mathrm{NA} \mathrm{Ph} 2$ objective with the 434 correction collar set to image through plastic, as previously described ${ }^{2}$. Illumination was provided by an 435 LED white-light system (Laser2000, Kettering, UK) in combination with filter cubes GFP-1828A-ZHE 436 (Semrock, NY, USA), YFP-2427B-ZHE (Semrock, NY, USA) and Filter Set 45 (Carl Zeiss Microscopy Ltd. 437 Cambridge, UK) used for GFP, YFP and RFP respectively. Emitted light was recorded using a back438 illuminated iXon888 Ultra EMCCD (Andor, UK) and images were processed using $\mathrm{FIII}^{39}$.

439 Multi-photon time-lapse imaging of gastruloids. Gastruloids were embedded in $10-100 \%$ Matrigel in 440 24-well plates (Sigma, EP0030741021 or M9312) at $96 \mathrm{~h}$ as described above, and imaged immediately 441 following embedding at $37{ }^{\circ} \mathrm{C}, 5 \% \mathrm{CO}_{2}$ with humidified air influx on a Leica SP8 multi-photon microscope 442 system using an HC PL APO 20x/0.75 air CS2 objective, a Coherent Chameleon Vision-S multi-photon 443 laser tuned to $960 \mathrm{~nm}$ and the pinhole maximally opened. The brightfield channel was recorded using a $444488 \mathrm{~nm}$ laser set at low intensity in combination with a transmission PMT. A z-stack of around 4 images 445 with a z-interval of $15 \mu \mathrm{m}$ was taken every $15 \mathrm{~min}$ (10 images per stack and at 12 min interval (Fig. 3c)) 446 for each individual gastruloid (frame accumulation 2 times, pixel dwell time $2.425 \mu \mathrm{s}$ ). Photons with a 447 wavelength between 505-555 $\mathrm{nm}$, and 555-680 nm were collected with two separate hybrid detectors and 448 assigned to a 16-bit pixel range. Alternatively, in Extended data Fig. 7d, a $514 \mathrm{~nm}$ solid state laser was 449 used during which photons were collected with a wavelength between 524-575 nm, and 600-700 nm with 
two separate hybrid detectors and assigned to a 16-bit pixel range. In this case the brightfield channel was recorded simultaneously with the other channels using a transmission PMT.

452 Treatment of Matrigel-embedded gastruloids with inhibitors. Gastruloids were embedded in 10-100\% 453 Matrigel at 96 as described above, and real-time imaging was started immediately after embedding. After 454 recording at least 2 timepoints and at most 4 timepoints for each replicate ( 30-60 min in total) the 455 microscope was paused and inhibitors were added without removing the culturing plate from the stage. 456 DAPT (Sigma, D5942; stock 10 mM in DMSO; used at 27 MM); PD0325901 (Sigma, PZ0162; stock 10 $457 \mathrm{mM}$ in DMSO); BGJ398 (Selleckchem, S2183; stock $1 \mathrm{mM}$ in DMSO; used at $0.2 \mu \mathrm{M}$ ); PD173074 458 (Peprotech, 2191178; stock 10mM in DMSO; used at $0.5 \mu \mathrm{M}$ ); FGF1 (Peprotech, 100-17A; stock 10 $459 \mu \mathrm{g} / \mathrm{mL}$ in $\mathrm{H}_{2} \mathrm{O}$; used at $0.02 \mu \mathrm{g} / \mathrm{mL}$ ); FGF10 (Peprotech, 100-26; stock $100 \mu \mathrm{g} / \mathrm{mL}$ in $\mathrm{H}_{2} \mathrm{O}$; used at 0.2 $460 \mu \mathrm{g} / \mathrm{mL}$ ); Chiron (CHI99021; Sigma, SML1046; stock $10 \mathrm{mM}$ in DMSO; used at $10 \mu \mathrm{M}$ ); IWP-2 (Sigma, 461 I0536; stock $2 \mathrm{mM}$ in DMSO; used at $2 \mu \mathrm{M}$ ); IWR-1 (Sigma, I0161; stock $10 \mathrm{mM}$ in DMSO; used at 10 $\mu \mathrm{M}$ ); LDN193189 (Sigma, SML0559; stock $0.1 \mathrm{mM}$ in $\mathrm{H}_{2} \mathrm{O}$; used at $0.2 \mu \mathrm{M}$ ).

463 Analysis of multi-photon time-lapse imaging data. Image analysis was done similar to previously described image-analysis methods ${ }^{15,23}$. Time-lapse imaging data was analysed using the ImageJ data processing package $\mathrm{FIJI}^{39}$. To filter out autofluorescence, the first channel (555-680 nm) was multiplied by 0.3 and subtracted from the second channel $(505-555 \mathrm{~nm})$. Then, a sum projection of all z-slices was generated for all timepoints. The resulting image was convolved using a gaussian filter with a sigma value of $1 \mu \mathrm{m}$. Kymographs were generated using the plug-in KymoResliceWide (https://github.com/ekatrukha/KymoResliceWide) by tracing the path of the differentiation front as it moves along the AP axis with a segmented line (60 pixels wide) and then blurred using a gaussian filter with a sigma value of 1 pixels. The intensity profile of the oscillations was measured at a constant distance from the differentiation front (dashed white line Fig. 2c) on the kymograph. The intensity profile of the oscillations was decomposed into a trend- and a cycle-component using Hodrick-Prescott filtering with an I of 800. Trend and cycle component for all replicates are shown in Extended Data Fig. 8. To make an estimation of the period of the Lfng oscillations, Lomb-Scargle analysis was performed with the maximum scanned frequency at half the temporal resolution and over-sampling set to $3^{40}$. The speed of the differentiation front and the elongation speed of the gastruloid were measured by first drawing a line along the differentiation front or posterior tip of the gastruloid on the kymograph, respectively, and then measuring the angle, as explained in Extended Data Fig. 9a.

Sample fixation for stainings. For gastruloids grown in 100-50 \% Matrigel, the medium was removed 482 For gastruloids grown in 25-10\% Matrigel, the medium/Matrigel was not removed in the first washing step 483 with PBS. After fixation, all samples were washed 3 times for 5 min in PBS-Tween $(0.1 \%$ Tween-20 (v/v)) 484 and washed 3 times for $3 \mathrm{~min}$ in TBS-Tween $(0.1 \%$ Tween-20 (v/v)) before digesting for 4 min with 25 485 $\mu \mathrm{g} / \mathrm{mL}$ Proteinase-K in TBS-Tween. The samples were then rinsed briefly 3 times with $2 \mathrm{mg} / \mathrm{mL}$ Glycine in 
TBS-Tween20, washed with TBS-Tween once, refixed for $30 \mathrm{~min}$ in $4 \%$ PFA and $0.05 \%$ GA in PBS at room temperature and washed 3 times in TBS-Tween.

488 In situ hybridization. ISH was performed as described before ${ }^{3,15}$. Briefly, samples were incubated for 4-5 hours in hybridization mix (5 mg/ml torula RNA (Sigma, R6625), 50\% deionized formamide (Sigma, AM9342) (v/v), 1.33x SSC, 0.1\% BSA (w/v), $125 \mu \mathrm{g} / \mathrm{ml}$ Heparin (Sigma, H3393), $10 \mathrm{mM}$ EDTA $0.5 \mathrm{pH}=$ 8.0, $0.1 \%$ Tween $20(\mathrm{v} / \mathrm{v}))$ at $68^{\circ} \mathrm{C}$ followed by incubation overnight in $150 \mathrm{ng} / \mathrm{mL}$ DIG-labelled probe in hybridization mix at $68^{\circ} \mathrm{C}$. Carryover Matrigel that was still present degraded during this incubation step in most instances. The hybridization mix with the probe was pre-incubated for $10 \mathrm{~min}$ at $80^{\circ} \mathrm{C}$. Samples were then washed twice for $30 \mathrm{~min}$ in pre-heated hybridization mix at $68{ }^{\circ} \mathrm{C}, 4$ times for $20 \mathrm{~min}$ in preheated 2x SSC-Tween $(0.1 \%$ Tween-20 $(\mathrm{v} / \mathrm{v}))$ at $68^{\circ} \mathrm{C}$, allowed to cool down and washed twice for $5 \mathrm{~min}$ in MAB-Tween $(0.1 \%$ Tween-20 $(\mathrm{v} / \mathrm{v}))$ at room temperature. The samples were blocked for 1.5 hours in blocking buffer (10\% heat inactivated sheep serum (Sigma, S3772) (v/v) and 1\% BSA (w/v) in MABTween) at room temperature, incubated for 4-5 hours in blocking buffer containing 1:2,000 anti-DIG-AP antibody (Sigma, 11093274910) at room temperature and washed 5 times for 10 min followed by washing overnight in MAB-Tween. Finally, the samples were washed 3 times in TBS-Tween, washed 3 times for $10 \mathrm{~min}$ in AP-buffer (100 mM Tris- $\mathrm{HCl} \mathrm{pH} \mathrm{8.0,} 100 \mathrm{mM} \mathrm{NaCl}, 50 \mathrm{mM} \mathrm{MgCl}$, 0.1\% Tween-20), stained for several hours in $1 \mathrm{~mL}$ BM purple (Sigma, 11442074001), washed 3 times for 5 min in TBS-Tween and refixed in 4\% PFA/PBS for $20 \mathrm{~min}$ at room temperature.

Imaging of gastruloids stained with in situ hybridization. In situ samples were imaged on a Leica M165FC stereo microscope with DMC5400 digital camera (Fig. 3a, right panel) or using a Nikon SMZ800N microscope (Fig. 3a left two panels) in TBS-Tween. was performed as described previously ${ }^{21}$ using reagents from Molecular Instruments. Briefly, each condition (up to 100 gastruloids) was incubated in $200-500 \mu \mathrm{L}$ of probe hybridization buffer for 5 min at room temperature and $30 \mathrm{~min}$ at $37^{\circ} \mathrm{C}$ before incubation with $4 \mathrm{pM}$ of each probe stock in $200-500 \mu \mathrm{L}$ probe hybridization buffer for $12-16$ hours at $37^{\circ} \mathrm{C}$. Next, samples were washed $4 \mathrm{x}$ with $500 \mu \mathrm{L}$ probe wash buffer for $15 \mathrm{~min}$ at $37^{\circ} \mathrm{C}, 2 \mathrm{x}$ with $1 \mathrm{~mL} 5 \mathrm{x}$ SSC-Tween for $10 \mathrm{~min}$ at room temperature and $1 \mathrm{x}$ with 200-500 $\mu \mathrm{L}$ amplification buffer for $5 \mathrm{~min}$ at room temperature. The hairpin mixture was prepared by separately heating both $\mathrm{h} 1$ and $\mathrm{h} 2$ of each hairpin to $95^{\circ} \mathrm{C}$ for 90 seconds and incubating these at room

515 temperature for $30 \mathrm{~min}$ in the dark. All the hairpin mixtures were then added to $200-500 \mu \mathrm{L}$ amplification 516 buffer at a concentration of $48 \mathrm{pM}$, which was then added to the samples and incubated for 12-16 hours 517 at room temperature in the dark. Samples were then washed at least $2 x$ with $1 \mathrm{~mL}$ SSC-Tween for 30 min 518 before imaging. HCR probe design: Uncx4.1 (Accession NM_013702.3, hairpin B1); Tbx18 (Accession 519 NM_023814.4, hairpin B3); Ripply2 (Accession NM_001037907, hairpin B2); hairpin B1 was labelled with 520 Alexa 594 and B2 and B3 with Alexa 488.

521 Multi-photon microscopy of HCR-stained gastruloids. HCR stained samples were imaged in TBS-T 522 on a Leica SP8 multi-photon microscope system using an HC PL APO 20x/0.75 air CS2 objective, a 
523 Coherent Chameleon Vision-S multi-photon laser tuned to $810 \mathrm{~nm}$ for the Alexa-594 dye, a $488 \mathrm{~nm}$ OPS524 laser for the Alexa-488 dye and the pinhole maximally opened. A z-stack of around 30 images with a z525 interval of $5 \mu \mathrm{m}$ was taken with frame accumulation set to 4 . Photons with a wavelength between 505-555 $526 \mathrm{~nm}$, and 555-680 nm were collected with two separate hybrid detectors and assigned to a 16-bit pixel 527 range for the Alexa-594 channel; photons with a wavelength between 498-550 $\mathrm{nm}$ were collected with a 528 hybrid detector and assigned to a 16-bit pixel range for the Alexa-488 channel. The brightfield channel 529 was recorded simultaneously with the Alexa-488 channel using a transmission PMT detector.

530 HCR data analysis. HCR imaging data was analysed using the ImageJ data processing package $\mathrm{FIJI}^{39}$. 531 First, all the images in a single stack were aligned using the ImageJ plug-in Correlescence 532 (https://github.com/ekatrukha/Correlescence), after which a maximum projection was generated for the 533 fluorescence channels. The posterior region of gastruloids was identified visually (the anterior end of 534 gastruloids is darker than the posterior end), and confirmed with Ripply2 stainings. To plot the intensity 535 profile along the AP axis, a segmented line with a width of 100 pixels was drawn, and the intensity was 536 measured along this line. To measure the peak-to-peak distances in the Uncx4.1 intensity profiles, a 537 LOWESS smoother (0.002 span) was applied, after which the maximal values corresponding to the peaks 538 were selected in $\mathrm{R}$.

539 Somite-size measurements in embryos. Somite-sizes were measured in 10 somite-stage paraffin540 embedded mouse embryos that were sectioned with $6 \mu \mathrm{m}$ sections, stained using a standard 541 haematoxylin and eosin staining and imaged with a Leica dm 4000 b led microscope with Leica DFC450 542 camera that was size-calibrated using a microscope calibration slide (Pyser-SGI). Somite-sizes were next 543 measured using Fiji. Measurements were validated by comparing results to somite-sizes in the EMAP 544 eMouse Atlas Project (http://www.emouseatlas.org) ${ }^{41}$.

\section{Additional references (for methods section)}

25. Fehling, H. J. et al. Tracking mesoderm induction and its specification to the hemangioblast during embryonic stem cell differentiation. Development 130, 4217-4227 (2003).

26. Faunes, F. et al. A membrane-associated $\beta$-catenin/Oct4 complex correlates with ground-state pluripotency in mouse embryonic stem cells. Development 140, 1171-1183 (2013).

27. Ferrer-Vaquer, A. et al. A sensitive and bright single-cell resolution live imaging reporter of Wnt/ßcatenin signaling in the mouse. BMC Dev. Biol. 10, 121 (2010).

28. Papanayotou, C. et al. A novel nodal enhancer dependent on pluripotency factors and smad2/3 signaling conditions a regulatory switch during epiblast maturation. PLOS Biol. 12, e1001890 (2014).

29. Kalmar, T. et al. Regulated fluctuations in nanog expression mediate cell fate decisions in embryonic stem cells. PLoS Biol. 7, e1000149 (2009). 
30. Turner, D. A. et al. Wnt/ß-catenin and FGF signalling direct the specification and maintenance of a neuromesodermal axial progenitor in ensembles of mouse embryonic stem cells. Development 141, 4243-4253 (2014).

31. Turner, D. A., Rué, P., Mackenzie, J. P., Davies, E. \& Martinez Arias, A. Brachyury cooperates with Wnt/ß-catenin signalling to elicit primitive-streak-like behaviour in differentiating mouse embryonic stem cells. BMC Biol. 12, 63 (2014).

32. Turner, D. A., Trott, J., Hayward, P., Rué, P. \& Martinez Arias, A. An interplay between extracellular signalling and the dynamics of the exit from pluripotency drives cell fate decisions in mouse ES cells. Biol. Open 3, 614-626 (2014).

33. Muraro, M. J. et al. A single-cell transcriptome atlas of the human pancreas. Cell Syst 3, 385-394 (2016).

34. Grün, D. et al. Single-cell messenger RNA sequencing reveals rare intestinal cell types. Nature 525, 251-255 (2015).

35. Wolf, F. A., Angerer, P. \& Theis, F. J. SCANPY: large-scale single-cell gene expression data analysis. Genome Biology 19, (2018).

36. Johnson, W. E., Li, C. \& Rabinovic, A. Adjusting batch effects in microarray expression data using empirical Bayes methods. Biostatistics 8, 118-127 (2007).

37. Chakraborty, S., Datta, S. \& Datta, S. Surrogate variable analysis using partial least squares (SVAPLS) in gene expression studies. Bioinformatics 28, 799-806 (2012).

38. Traag, V. A., Waltman, L. \& van Eck, N. J. From Louvain to Leiden: guaranteeing well-connected communities. Sci. Rep. 9, 5233 (2019).

39. Schindelin, J. et al. Fiji: an open-source platform for biological-image analysis. Nat. Methods 9, 676682 (2012).

40. Glynn, E. F., Chen, J. \& Mushegian, A. R. Detecting periodic patterns in unevenly spaced gene expression time series using Lomb-Scargle periodograms. Bioinformatics 22, 310-316 (2006).

41. Richardson, L. et al. EMAGE mouse embryo spatial gene expression database: 2014 update. Nucleic Acids Res. 42, D835-44 (2014).

\section{Extended Data Figure legends}


Extended Data Fig. 1 | scRNA-seq on $120 \mathrm{~h}$ mouse gastruloids and comparison to embryos. a, Fluorescence-activated cell sorting (FACS) gating strategy prior to scRNA-seq. Live cells were selected based on DAPI staining. Four sequential gates (P1-P4) were used; cells from gate P4 were used for scRNA- seq. SSC, side scatter; FSC, forward scatter; $H$, height; W, width; $A$, area. b, Box plot showing the median number of transcripts (left) and genes (right) detected per cell for SORT-seq experiments on E14-IB10 (E14-S) and LfngT2AVenus gastruloids (Lfng-S), and for 10x Genomics experiments on LfngT2AVenus gastruloids (Lfng-10x). The box extends from the lower to the upper quartile. Whiskers, $1.5 x$ interquartile range; flier points are those past the end of the whiskers. c, Uniform manifold approximation and projection (UMAP) plot for each experiment separately. Colour of each cell is the same as the colour of that particular cell in Fig. 1a. d, UMAP obtained by analysing all the cells from the different experiments together, where cells are coloured according to their batch (Methods, Supplementary Table 1). The black line indicates the symmetry line in clusters 1-8 used to generate the linearized UMAP in Fig. 1c (Methods). e, Fraction of E14-IB10 and LfngT2AVenus cells in each scRNAseq cluster from Fig. 1a. Blue, green and black numbers, number of E14-IB10, LfngT2AVenus and total cells in each cluster (Supplementary Tables 1, 4). f, Fraction of cells for each cell type in each plate in SORT-seq experiments (Lfng-S, E14-S), and in each experimental batch in 10x Genomics experiments (Lfng-10x). Box plots: center line, median; box limits, 1st and 3rd quartiles; whiskers, range. g, Fraction of cells detected in the E8.5 mouse embryo scRNA-seq dataset ${ }^{4}$ used to compare our gastruloid scRNA-seq data with. Exact numbers in each cluster are indicated. $\mathbf{h}$, Dot plot showing overlapping genes between significantly upregulated genes for each gastruloid scRNA-seq cluster (Supplementary Table 2), and upregulated genes for each E7.0-E8.5 mouse embryonic cell type ${ }^{4}$. Dot colour indicates the probability of finding such a number of overlapping genes between the two sets by random chance (P-value, Methods), and dot size represents the number of overlapping genes. Blue colouring, embryonic stage. E14, E14IB10; Lfng, LfngT2AVenus; S, SORT-seq ${ }^{33}$; 10x, 10x Genomics; MD, mesoderm; EcD, ectoderm; NMP, neuro-mesodermal progenitors; ExE, extra-embryonic; EnD, endoderm; Haemato, haemato-endothelial; prog, progenitors; PGC, primordial germ cells; Ant, anterior; PSM, presomitic mesoderm.

Extended Data Fig. 2 | Expression of relevant markers in gastruloid scRNA-seq dataset. a, Mean log expression of relevant markers of outlier populations (clusters 9-13) plotted on the UMAP from Fig. 1a. Olfr129 and Onecut1, head mesenchyme (cluster 9); Etv2, haemato-endothelial progenitors (bottom part of cluster 10); Kdr, haemato-endothelial progenitors and endothelium (cluster 10); Cdh5 and Tie1, endothelium (top part of cluster 10); Tbx4, Hoxa11, Ass1 and Bmp7, allantois (cluster 11); Ephx2, Mt1,

578 Utf1 and Pou5f1, primordial germ cell like or extra-embryonic ectoderm (cluster 12); Col4a1, Epcam and 579 Sox17, endoderm (cluster 13). b, Mean log normalized expression of relevant markers of clusters 1-8 580 plotted on the UMAP from Fig. 1a. Hand2 and Gata6, heart (cluster 1); Meox2 and Pax3, differentiated somite (cluster 3); Aldh1a2 and Uncx4.1, somite (cluster 4); Lfng, Mesp2, Ripply2 and DII1, differentiation front (cluster 5); Hes7 and Tbx6, presomitic mesoderm (cluster 6); Wnt3a, Fgf17, Fgf8, Cyp26a1, Nkx1-2 
and $T$, tail bud containing neuro-mesodermal progenitors (cluster 7); Pax6, Sox1, Hes3 and Sox2, differentiated neural cells (spinal cord; cluster 8). Expression was first count-normalized to 10,000 for each cell (Methods), and then log-transformed. Additional markers of all clusters are provided in Supplementary Table 2.

Extended Data Fig. 3 | Number of genes and reads in gastruloid and embryo tomo-seq datasets, and comparison to microscopy data. a-c, Number of unique transcripts and genes detected in 3 E14IB10 $120 \mathrm{~h}$ mouse gastruloids that were sectioned using $20 \mu \mathrm{m}$ sections and $2 \mathrm{E} 14-\mathrm{lb} 10120 \mathrm{~h}$ mouse gastruloids that were sectioned using $8 \mu \mathrm{m}$ sections (a); in 3 LfngT2AVenus $120 \mathrm{~h}$ mouse gastruloids that were sectioned using $20 \mu \mathrm{m}$ sections (b); and in 3 E8.5 mouse embryos that were sectioned using $20 \mu \mathrm{m}$ sections (c). Due to their length, embryo sections were collected in two sequential 96-well plates. d, Validation of tomo-seq data with microscopy. Top panels, Brachyury ${ }^{G F P}$, Wnt signalling activity (as reported using a TCF/LEF ${ }^{\text {mCherry }}$ mouse ESC line) and Nodal ${ }^{\text {YFP }}$ expression in $120 \mathrm{~h}$ mouse gastruloids as measured by microscopy (Methods). Barplots showing the normalized expression levels of Brachyury, Wnt3a and Nodal in 120 h E14-IB10 gastruloids, 120 h LfngT2AVenus gastruloids and E8.5 mouse embryos as determined by tomo-seq (Methods), and in the posterior mesoderm of E9.5 mouse embryos as determined by microarray ${ }^{12}$. e, Scaled average z-score of significantly upregulated genes detected in each single cell cluster from Fig. 1a (Supplementary Table 2) as measured in the averaged LfngT2AVenus tomo-seq gastruloid. Scale bar, $100 \mu \mathrm{m}$; A, anterior; $\mathrm{P}$, posterior.

Extended Data Fig. 4 | Individual replicates of gastruloids, E8.5 embryo tomo-seq and E9.5 posterior mesoderm datasets, and comparison to gastruloid and E8.5 embryonic scRNA-seq datasets. a, Heatmaps showing the AP expression patterns of 1,199 genes as detected by tomo-seq ${ }^{11}$ in individual replicates of 120 h E14-IB10 gastruloids ( $n=3$ gastruloids, $20 \mu \mathrm{m}$ sections and $\mathrm{n}=2$ gastruloids, $8 \mu \mathrm{m}$ sections) that were cultured in standard ${ }^{1,20}$ (non-Matrigel based) conditions; average

608 heatmap of the 5 replicates; average expression of genes found in each tomo-seq domain in the E14IB10 tomo-seq dataset, projected in the UMAP from Fig. 1a; dot plot showing overlapping genes between genes detected in each tomo-seq domain in the E14-IB10 tomo-seq dataset, and upregulated genes for each E8.5 mouse embryonic cell type ${ }^{4}$. Dot colour represents the probability of finding such a number of overlapping genes between the two sets by random chance (Methods), and dot size represents the number of overlapping genes. Only genes that were reproducible between all replicates are shown (Methods). Genes are clustered based on their AP expression pattern (Methods); Roman-numbered bars represent tomo-seq clusters. b, Similar to panel a, but for 1,456 genes in 120 h LfngT2AVenus ${ }^{15}$ ( $n=3$

616 gastruloids; $20 \mu \mathrm{m}$ sections) gastruloids that were cultured in standard ${ }^{1,20}$ (non-Matrigel based) 617 conditions. c, Similar to panel a, but for 1,553 genes in E8.5 embryos ( $n=3$ embryos, $20 \mu m$ sections). d, 618 Similar to panel a, but for 1,989 genes in an E9.5 mouse embryo posterior mesoderm dataset (tail bud to 619 newly formed somite; $n=3$ embryos; previously published microarray data; $\sim 100 \mu \mathrm{m}$ sections ${ }^{12}$. All genes 
are in Supplementary Table 6. E14, E14-IB10; Lfng, LfngT2AVenus; AP, anterior-posterior; MD, mesoderm; NMP, neuro-mesodermal progenitors; EcD, ectoderm; Def, definitive; EnD, endoderm; Haemato, haemato-endothelial; prog, progenitors; ExE, extra-embryonic; FMH, fore- mid - hindbrain.

Extended Data Fig. 5 | Comparisons between mouse gastruloid and mouse embryo datasets, including genes that are reproducible in at least one system. a, Heatmap showing the average AP expression pattern of 2,065 genes as detected by tomo-seq ${ }^{11}$ in $120 \mathrm{~h}$ mouse gastruloids that were generated from E14-IB10 and LfngT2AVenus ${ }^{15}$ mouse ESCs and that were cultured in standard, ${ }^{1,20}$ (nonMatrigel based) conditions; average expression of genes found in each tomo-seq domain in the E14IB10- LfngT2AVenus comparison heatmap, projected in the UMAP from Fig. 1a; dot plot showing overlapping genes between genes detected in each tomo-seq domain in panel a, and upregulated genes for E8.5 mouse embryonic cell types ${ }^{4}$. Dot colour represents the probability of finding such a number of overlapping genes by random chance (Methods), and dot size represents the number of overlapping genes. In contrast to the heatmaps in Fig. 1, this heatmap contains genes that were reproducible in either E14-IB10 ( $n=3$ gastruloids, $20 \mu$ m sections and $n=2$ gastruloids, $8 \mu$ m sections) or LfngT2AVenus $(n=$ 3 gastruloids; $20 \mu \mathrm{m}$ sections) gastruloids (Methods, Supplementary Tables 5-6, Extended Data Fig. 4). This means that genes that are reproducible in E14-IB10 replicates but not in LfngT2AVenus replicates, and vice versa, are included. Genes are clustered based on their AP expression pattern (Methods); Roman-numbered bars represent tomo-seq clusters, which are also indicated with the gray-black barplot. The red-to-white barplots indicate the $P$-value of reproducibility of each gene in each heatmap. The order of these barplots correspond to the order of the heatmaps. b, Similar to panel a, but for 2,804 genes in that were reproducible in E14-IB10 ( $n=3$ gastruloids, $20 \mu \mathrm{m}$ sections and $\mathrm{n}=2$ gastruloids, $8 \mu \mathrm{m}$ sections) or LfngT2AVenus ( $n=3$ gastruloids; $20 \mu \mathrm{m}$ sections) or E8.5 mouse embryos ( $n=3$ embryos; $20 \mu \mathrm{m}$ sections). c, Similar to panel a, but for 3,086 genes in that were reproducible in E14-IB10 ( $\mathrm{n}=3$ gastruloids, $20 \mu \mathrm{m}$ sections and $\mathrm{n}=2$ gastruloids, $8 \mu \mathrm{m}$ sections) or LfngT2AVenus ( $\mathrm{n}=3$ gastruloids; 20

$645 \mu \mathrm{m}$ sections) or the E9.5 mouse embryo posterior mesoderm dataset (tail bud to newly formed somite; $\mathrm{n}$

$646=3$ embryos; previously published microarray data; $\sim 100 \mu \mathrm{m}$ sections ${ }^{12}$. Here, only the first 15 tomo-seq clusters are projected onto the UMAPs. Gene lists are provided in Supplementary Table 8. E14, E14648 IB10; Lfng, LfngT2AVenus; AP, anterior-posterior; MD, mesoderm; NMP, neuro-mesodermal progenitors; $649 \mathrm{EnD}$, endoderm; Haemato, haemato-endothelial; prog, progenitors; PGC, primordial germ cells; EcD, ectoderm; Def, definitive; ExE, extra-embryonic.

652 Extended Data Fig. 6 | Gene expression profiles in gastruloid and embryo tomo-seq datasets. 653 Lineplots for the normalized AP expression of genes emphasized in Fig. 1d, $f$ and $g$ for the E14-IB10 and 654 LfngT2AVenus gastruloids, and for the E8.5 mouse embryo, as measured by tomo-seq ${ }^{11}$. Each color is a 655 different replicate. 

gastruloids that were embedded in $100 \%$ Matrigel at $96 \mathrm{~h}$. a-d, Kymographs (space-time plots) of brightfield channel and LfngT2AVenus signal along the AP axis of all replicates from all time-lapse experiments (Experiments 1-4) that are presented in Fig. $2 f$ and in Extended Data Fig. 8e,f. These gastruloids were embedded in $100 \%$ Matrigel (Methods) to stabilize them during imaging, and subsequently imaged for at least 17 hours (Supplementary Video 1-2, 4-5). Inhibitors were added at the start of the time-lapse (Methods) and are indicated above the kymographs, together with their concentration. Asterisks refer to gastruloids used to generate Fig. 3e and Extended Data Fig. 8b. e, Realtime imaging of a LfngT2AVenus gastruloid that was embedded in $100 \%$ Matrigel at $96 \mathrm{~h}$ and to which the Notch-inhibitor DAPT was added at $96.5 \mathrm{~h}$ (Supplementary Video 2; Lfng signal disappears $\sim 6$ hours after DAPT addition). Corresponding kymographs in panel a. A, Anterior; P, Posterior.

Extended Data Fig. 8 | Detrending procedure and Lomb-Scargle analysis of replicates from Fig. 2, and measurements of elongation and differentiation front speed in small panel screening and upon BGJ389 and PD17 treatment. a, Black line, measured intensity of the Lfng signal along the whitedashed line in Fig. 2c; blue line, trend (Methods) of this signal, and periodogram of the Lfng oscillations in Fig. 2d, as determined by Lomb-Scargle decomposition. b, As in a, but then for the 13 DMSO-control LfngT2AVenus gastruloid replicates shown in Extended Data Fig. 7c-d. c, cyclical component of the scaled intensity of the LfngT2AVenus oscillations relative to the trendline shown in b. A.U., arbitrary units. d, Periodogram of the Lfng oscillations in c, as determined by Lomb-Scargle decomposition (Methods). Gastruloids used for this experiment were embedded in $100 \%$ Matrigel at $96 \mathrm{~h}$, and subsequently imaged for at least 17 hours (Supplementary Video 6). e-f, Speed of posterior gastruloid elongation ( $\left.V_{P S M}\right)$ and speed of posteriorly moving differentiation front ( $\mathrm{V}_{\mathrm{DIFF}}$; see explanation in Extended Data Fig. 9a) in LfngT2AVenus gastruloids treated with DMSO (control), or with various inhibitors (Supplementary Videos $3,5)$. Points refer to replicates; kymographs of replicates in Extended Data Fig. 7. Box plots: center line, median; box limits, 1st and 3rd quartiles; whiskers, range.

Extended Data Fig. 9 | Explanation on how elongation and differentiation front speed were measured, and HCR stainings and live-imaging kymographs of gastruloids embedded in $10 \%$ Matrigel. a, Kymographs (space-time plots) of brightfield channel and LfngT2AVenus signal along the AP axis of a DMSO-treated (control) and a PD03-treated (MEK/ERK inhibitor) LfngT2AVenus gastruloid. 688 Gastruloids were embedded in $100 \%$ Matrigel at $96 \mathrm{~h}$; DMSO or PD03 (66.7 $\mu \mathrm{M})$ was added at $96.5 \mathrm{~h}$. 689 Kymographs were used to measure the elongation speed of the gastruloid (angle of blue dashed line; $690 \mathrm{~V}_{\mathrm{PSM}}$; Methods) and the speed of the differentiation front (angle of red dashed line; $V_{\mathrm{DIFF}}$ ). b, 691 LfngT2AVenus gastruloids that were embedded in 10\% Matrigel (Methods) at $96 \mathrm{~h}$ and stained for 692 Uncx4.1 using $\mathrm{HCR}^{21}$ at 120 h. Zoom in of the left gastruloid is shown in Fig. 3b. c, Kymographs of 693 LfngT2AVenus signal and brightfield channel along the AP axis of gastruloids that were embedded in 
$69410 \%$ Matrigel at $96 \mathrm{~h}$, and subsequently imaged for 20 hours (Supplementary Video 6). Top kymograph 695 belongs to the gastruloid that is shown in Fig. 3c. A, Anterior; P, Posterior; Scale bar, $200 \mu \mathrm{m}$.

696 Extended Data Fig. 10 | Uncx4.1/Tbx18IRipply2 stainings and somite size measurements. a, HCR ${ }^{21}$ 697 double staining for Uncx4.1 (cyan) and Tbx18 (magenta) on a $120 \mathrm{~h}$ LfngT2AVenus gastruloids 698 embedded in $10 \%$ Matrigel at $96 \mathrm{~h}$. To replicate 4, $1.3 \mu \mathrm{M}$ of PD03 was added at $96.5 \mathrm{~h}$. b, Similar to 699 panel a, but now for Uncx4.1 (cyan) and Ripply2 (yellow). c, Intensity of Uncx4.1 and Tbx18 signal along 700 the AP axis of the gastruloids in panel a. Peaks (circles) are called on the smoothened Uncx4.1 profile 701 (dark blue; Methods). d, Similar to panel c, but now for the Uncx4.1 and Ripply2 stained gastruloids from 702 panel b. e, Distance between Uncx4.1 peaks in the $120 \mathrm{~h}$ LfngT2AVenus gastruloids $(n=7)$ from 703 replicates 1-6 in panels a-d and in replicate 7 (which is shown in Fig. 3d). Replicate 8 was excluded from 704 quantification and both replicate 4 and 7 were incubated in $1.3 \mu \mathrm{M}$ PD03 from $96-120 \mathrm{~h}$. A, Anterior; P, 705 Posterior; Scale bar, $200 \mu \mathrm{m}$. 